\title{
Towards Complete Resolution of DNA/Carbon Nanotube Hybrids by Aqueous Two-phase Systems
}

\author{
Min Lyu ${ }^{1}$, Brendan Meany ${ }^{2}$, Juan Yang ${ }^{1^{*}}$, Yan $\mathrm{Li}^{1^{*}}$, Ming Zheng ${ }^{2^{*}}$ \\ ${ }^{1}$ Beijing National Laboratory for Molecular Science, Key Laboratory for the Physics and Chemistry of \\ Nanodevices, State Key Laboratory of Rare Earth Materials Chemistry and Applications, College of Chemistry \\ and Molecular Engineering, Peking University, Beijing 100871, China \\ ${ }^{2}$ Materials Science and Engineering Division, National Institute of Standards and Technology, 100 Bureau \\ Drive, Gaithersburg, Maryland 20899, United States
}

Certain equipment, instruments or materials are identified in this paper in order to adequately specify the experimental details. Such identification does not imply recommendation by the National Institute of Standards and Technology (NIST) nor does it imply the materials are necessarily the best available for the purpose. Where provided, uncertainty is reported as one standard deviation.

\section{Exploration of ATP Systems}

Scheme S1 exhibits a schematic phase diagram of ATP system formed by PEG and DX. In a solution containing PEG, DX and water, at low concentrations of polymers, only one phase (purple area) exist in the solution. When the concentrations of PEG and DX are above certain level, phase separation occurs (white area). The boundary between the single-phase region and the two-phase region is the binodal or coexistence curve. Closer to the curve, the system is more sensitive to a small change in composition. A small change of the composition from below to above the binodal curve, the system changes from one phase to two phases, and vice versa. The hollow dot $\mathrm{K}$ on the curve is the critical point and the composition represented by $\mathrm{K}$ is the critical composition. Tie lines, such as BT and B1T1, which connect the two points on the binodal curve, are shown in the phase diagram. The end point B and B1 represent the composition of both polymers in the top phase, while $\mathrm{T}$ and $\mathrm{T} 1$ represent the composition of the bottom phase. Thus all the points on a same tie line (such as point A, A' and A') will have the same top phase and bottom phase composition but different top to bottom volume ratio. For an ATP system whose composition is represented by point A, its top and bottom's composition is represented by point $\mathrm{T}$ and $\mathrm{B}$ and its top to bottom volume ratio is $\mathrm{AB} / \mathrm{AT}$.

According to those properties described above, one can explore an ATP system without knowing its phase diagram. Take the exploration of PEG1.5kDa/DX250kDa ATP system as an example. First, $60 \mathrm{wt} \%$ PEG1.5kDa and 20 wt \% DX250kDa stock solutions are prepared. By mixing $100 \mu \mathrm{L} 20$ wt \% Dextran250kDa, 20 $\mu \mathrm{L} 60$ wt \% PEG1.5kDa, phase separation occurs, yielding a Vt (volume of top phase)/Vb (volume of bottom phase) close to 1. Since this system is too viscous and far away from the critical point, $20 \mu \mathrm{L}$ water is added to dilute the system, moving it from point $\mathrm{A}$ to $\mathrm{A} 1$, and $\mathrm{Vt} / \mathrm{Vb}$ from 1 to around $1 / 2$. This informs us that further dilution of the initial composition 
A will reduce the volume of the top phase. In order to prepare an ATP system close to the critical point and with an almost equal top and bottom volume, we need to move the composition upwards in the phase diagram, which means the concentration of PEG1.5kDa must be increased. So, another $2 \mu \mathrm{L} 60 \mathrm{wt} \%$ PEG1.5kDa is added into the system, which moves the composition of the system from $\mathrm{A} 1$ to $\mathrm{A} 2$ and the $\mathrm{Vt} / \mathrm{Vb}$ back to around 1 . Then, keep on diluting the system and adding more PEG1.5kDa to tune the $\mathrm{Vt} / \mathrm{Vb}$ back to 1 . With few more iterations, it's possible for one to approach the critical point $\mathrm{K}$. However, for practical usage, it's better to keep away from the critical point a little bit. Because, in a real separation experiment, some modulating agents are needed to add into the system. In this way, we can reserve some space for introducing modulating agents. In the end, the best composition of the PEG1.5kDa/Dextran250kDa ATP system (10:0) we found is $8.57 \mathrm{wt} \%$ PEG1.5kDa and $10.1 \mathrm{wt} \%$ Dextran250kDa.
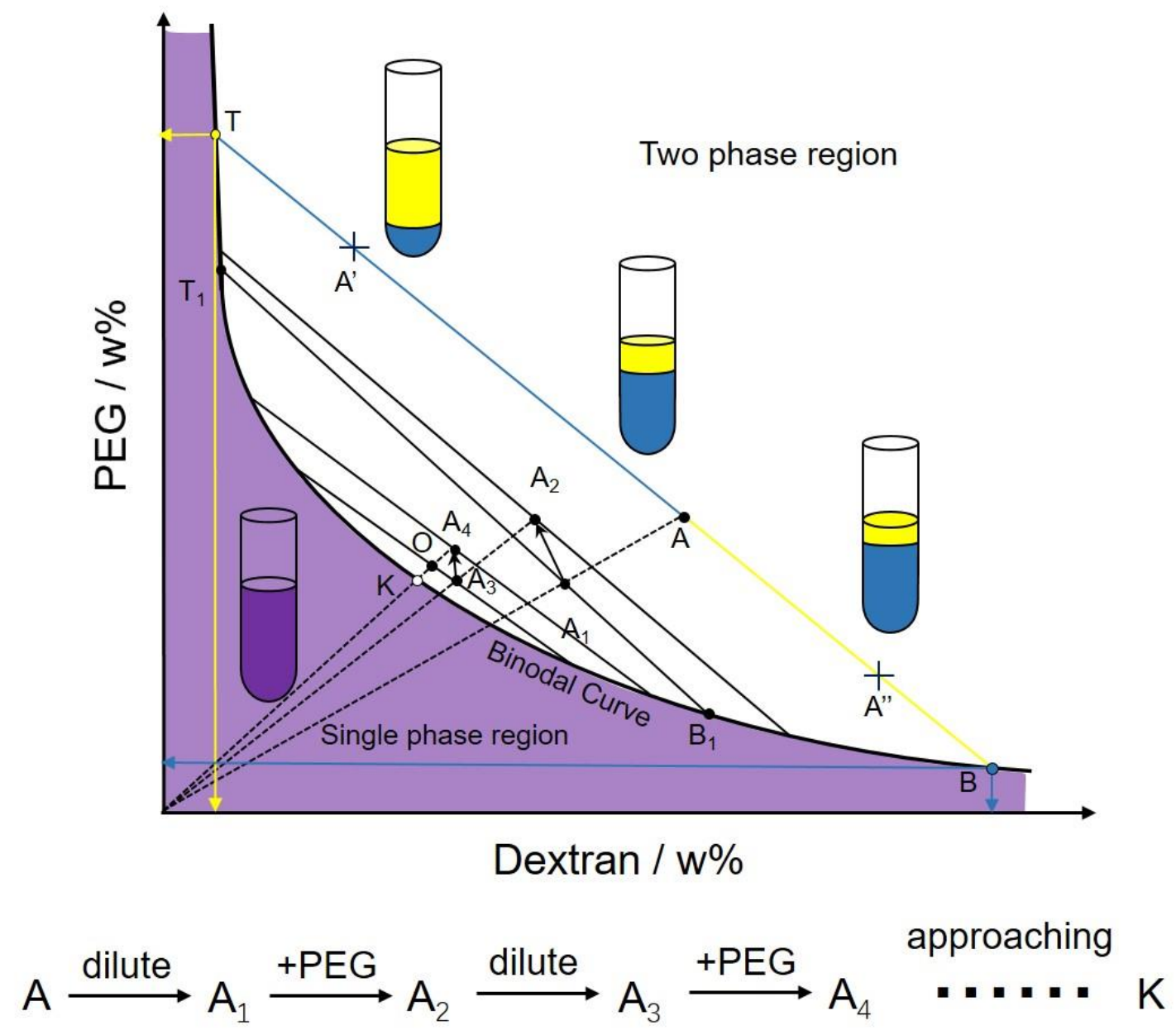

Scheme S1 Exploration of PEG/DX ATP system

Two enantiomers of $(6,5)$ and $(8,3)$ Purified in PEG/PAM ATP System 

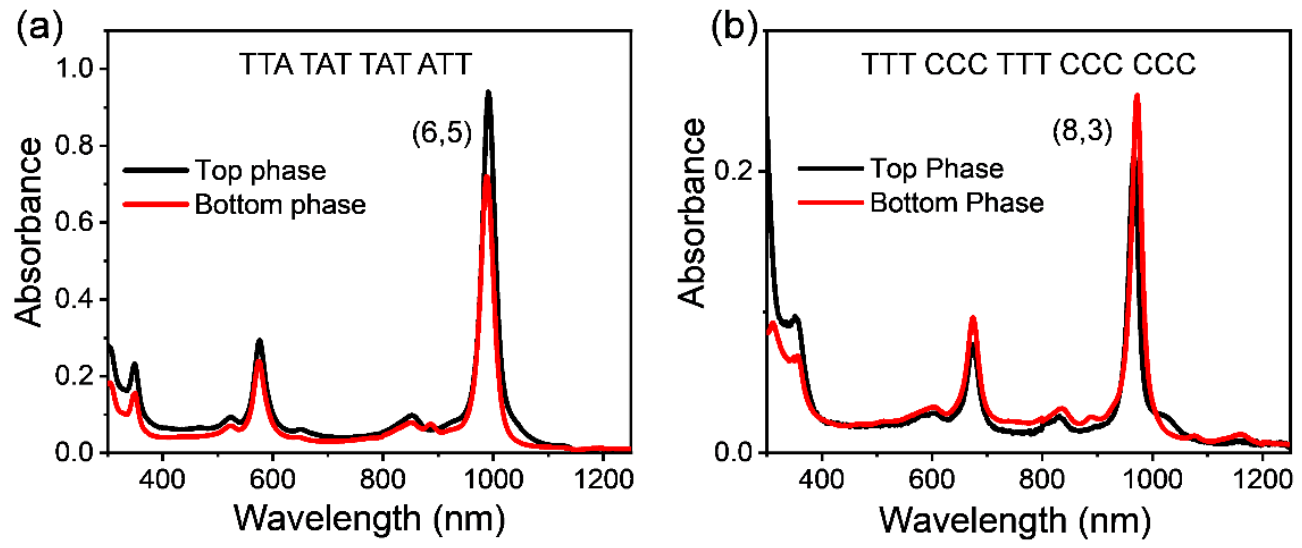

Figure S1. Absorption spectrum of (a) two $(6,5)$ enantiomers obtained from the $(6,5)$ super sequence in PEG/PAM ATP system. (b) two $(8,3)$ enantiomers obtained from the $(8,3)$ super sequence in PEG/PAM ATP system. The corresponding super sequence is shown in the graph.

After the TTA TAT TAT ATT-SG65i dispersion was loaded in PEG/PAM ATP system, the top phase is highly enriched in (-) 6,5 enantiomer while most of the SWCNTs partition into the bottom phase. Then fresh top phase and PVP are sequentially added to allow the extraction of the intermediate top fractions. The SWCNTs remaining in the bottom phase is (+) 6,5 enantiomer. After the TTT CCC TTT CCC CCC-SG65i dispersion was loaded in PEG/PAM ATP system, the top phase is highly enriched in one kind of $(8,3)$ enantiomer while most of the SWCNTs partition into the bottom phase. Then fresh top phase and PVP are sequentially added to allow the extraction of the intermediate top fractions. The SWCNTs remaining in the bottom phase is another kind of $(8,3)$ enantiomer. The E11 transition peak wavelength of $(6,5)$ and $(8,3)$ from the top and bottom phase is slightly different. The Circular Dichroism (CD) spectra of these two $(6,5)$ enantiomers are provided in the previous paper. ${ }^{1}$ We did not measure the CD spectra of the two kinds of $(8,3)$ yet, but we speculate that the two kinds of $(8,3)$ obtained are enantiomers.

\section{Preparation of PEG/DX ATP Systems}

For any ATP system, we recommend preparing two concentrated polymer/water solutions before mixing. Then, the weight of two concentrated polymer/water solutions and water needed to make an ATP solution is calculated according to Table 1.

For PEG1.5kDa/DX250kDa ATP system, after the 10:0 composition of PEG1.5kDa/DX250kDa is determined, the $7: 3$ composition is calculated to be $12.2 \mathrm{wt} \%$ PEG1.5kDa and $14.4 \mathrm{wt} \% \mathrm{DX} 250 \mathrm{kDa}$.

In our experiment, we first prepare the 7:3 PEG1.5kDa/Dx250kDa ATP solution according to its composition by mixing $12.94 \mathrm{~g} 20 \mathrm{wt} \% \mathrm{DX} 250 \mathrm{kDa}, 3.676 \mathrm{~g} 60 \mathrm{wt} \%$ PEG1.5kDa and $1.855 \mathrm{~g}$ deionized water. Then 10:0 solution will be prepared by mixing 7 parts of 7:3 solution with 3 parts of water. Due to the high viscosity of the 7:3 solution, one need to vortex the solution violently and suck the cloudy solution out immediately after vortex. The 10:0 solution is incubated at room temperature $\left(20^{\circ} \mathrm{C}\right)$ overnight to allow phase separation. Then we extract the top and bottom phase separately and store them in the refrigerator (Bacterial will breed in this system if it is stored at room temperature for a long time).

\section{Screening the Best PEG/DX Combination}


Firstly, fixing the PEG molecular weight at $6 \mathrm{kDa}$, we test the sorting result of TTA TAT TAT ATT-SG65i dispersion (in $0.1 \mathrm{M} \mathrm{NaCl}$ ) and TTT CCC TTT CCC CCC-SG65i dispersion (in $0.1 \mathrm{M} \mathrm{NaCl}$ ) in PEG6kDa/DX40kDa, PEG6kDa/DX70kDa, PEG6kDa/DX250kDa and PEG6kDa/DX500kDa ATP systems. The absorbance spectra of the first top fraction and the final bottom fraction are shown in Figure S2. As the DX molecular weight increase from $40 \mathrm{kDa}$ to $250 \mathrm{kDa}$, the yield of the top phase increase at the expense of losing a little bit purity. However, the purity of the bottom phase increases a lot. When the DX molecular weight further increase to $500 \mathrm{kDa}$, the purity of top fractions is still reasonably good while the bottom fractions (especially the final bottom fraction for TTA TAT TAT ATT-SG65i dispersion) begin to deteriorate. In terms of both the yield and purity, we think the best choice is DX250kDa.
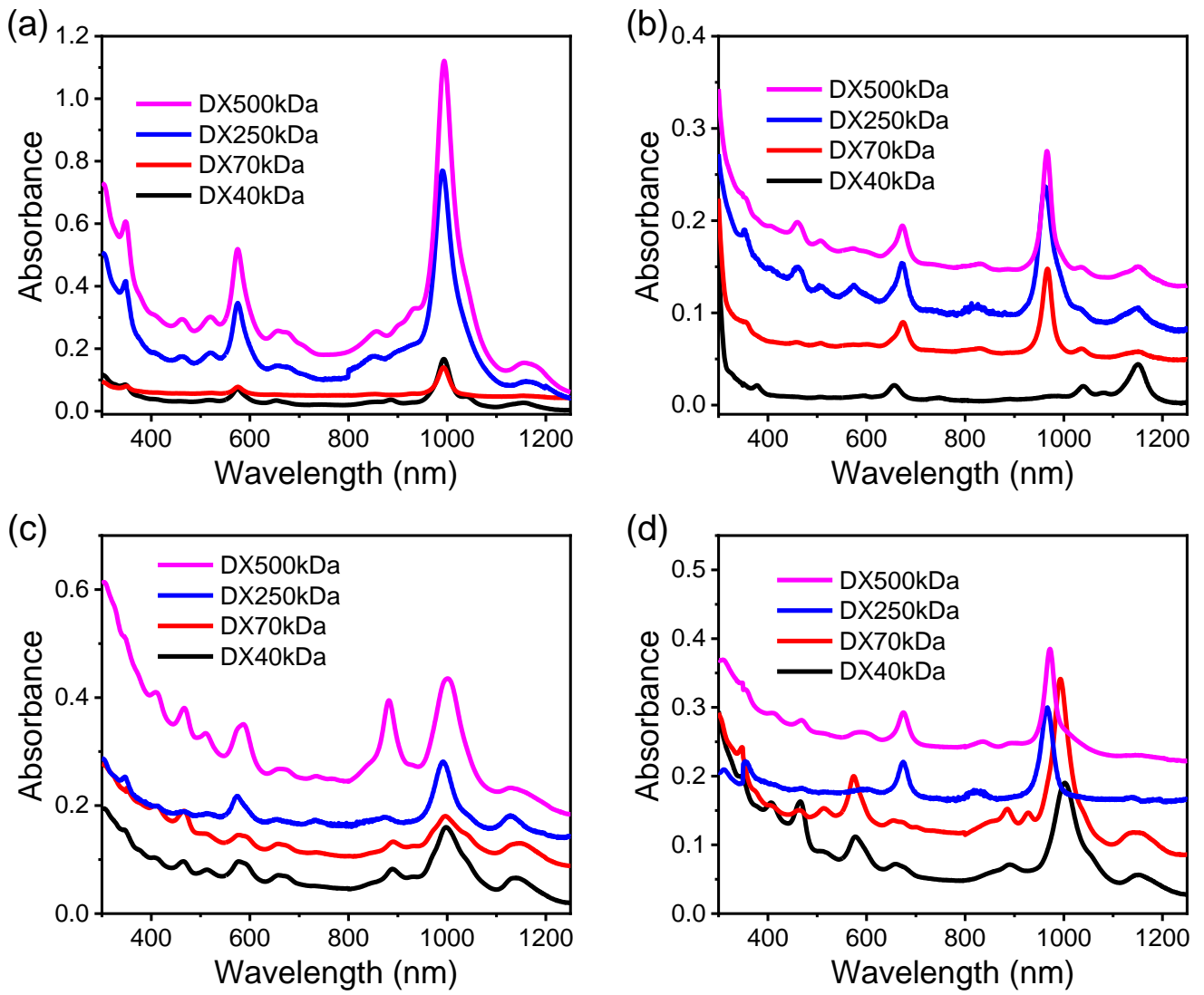

Figure S2. Absorption spectrum of the first top fraction obtained after loading (a) TTA TAT TAT ATT-SG65i (in $0.1 \mathrm{M} \mathrm{NaCl}$ ) and (b) TTT CCC TTT CCC CCC-SG65i (in $0.1 \mathrm{M} \mathrm{NaCl}$ ) in PEG6kDa/DX40kDa, PEG6kDa/DX70kDa, PEG6kDa/DX250kDa and PEG6kDa/DX500kDa ATP systems. Absorption spectrum of the final bottom fraction obtained after loading (c) TTA TAT TAT ATT-SG65i (in 0.1 M NaCl) and (d) TTT CCC TTT CCC CCC-SG65i (in 0.1 M NaCl) in PEG6kDa/DX40kDa, PEG6kDa/DX70kDa, PEG6kDa/DX250kDa and PEG6kDa/DX500kDaATP systems. 
Although not as obvious as the effect of DX MW, the MW of PEG also affects the partition behavior of SWCNT dispersion in PEG/Dextran ATP system. As Figure S3a and S3b shows, among 3 combinations formed by DX250kDa with PEG6kDa, PEG2kDa and PEG1.5kDa, the overall trend is that as the decrease of PEG MW, more $(6,5)$ and $(8,3)$ appears in the top phase. The best combination is PEG1.5kDa and DX250kDa. The absorption spectrum of two kinds of $(6,5)$ and $(8,3)$ obtained from the top and bottom phase are shown in Figure S3e and S3f.

(a)

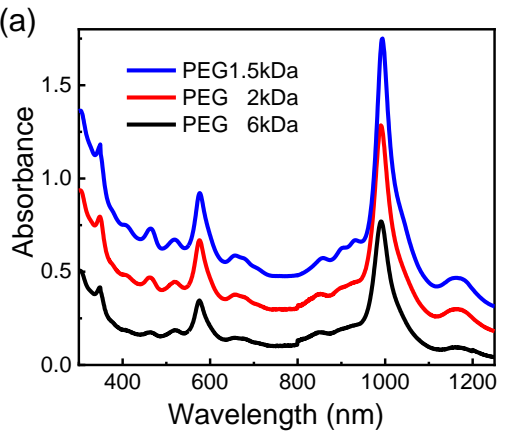

(c)
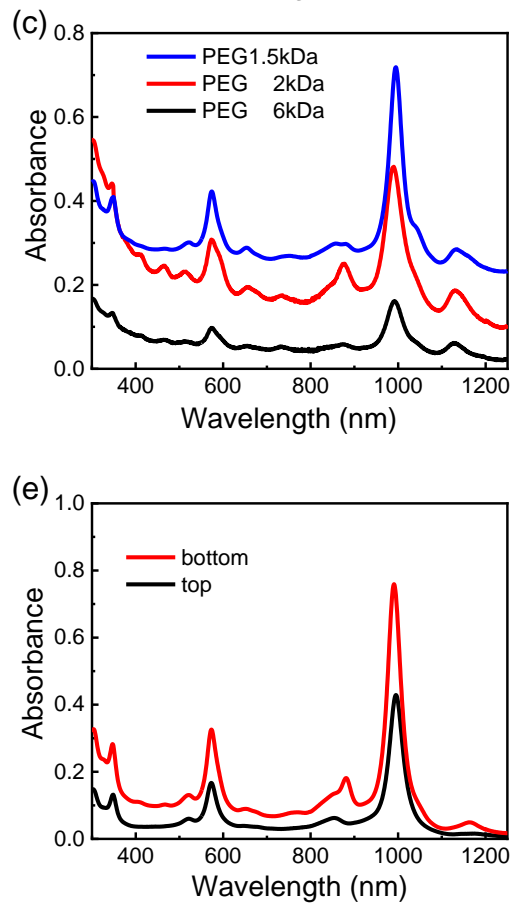

(b)

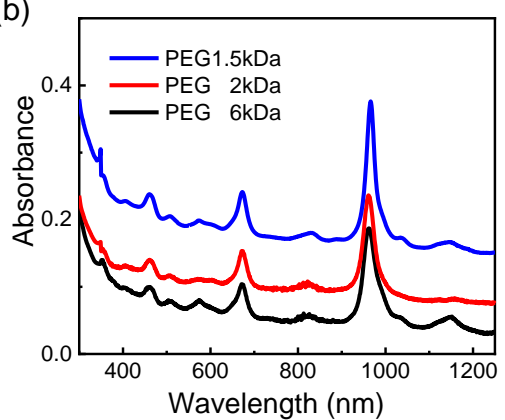

(d)
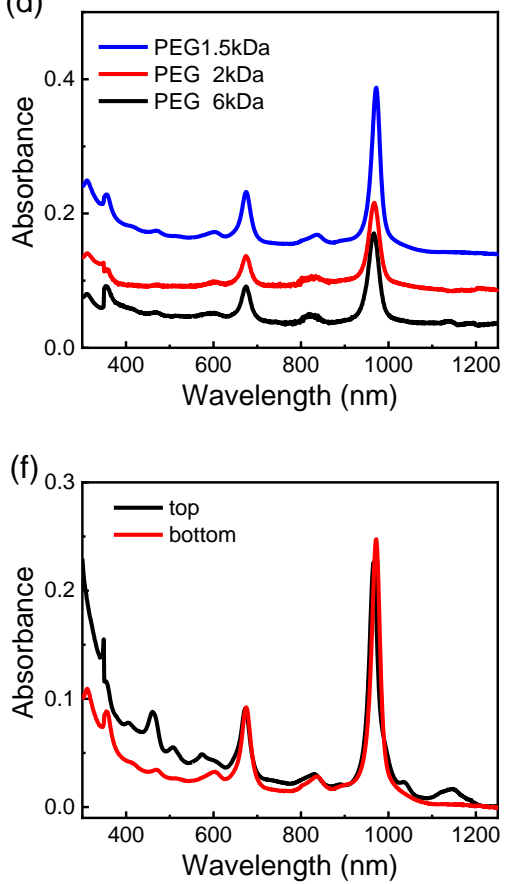

Figure S3. Absorption spectrum of the first top fraction obtained after loading (a) TTA TAT TAT ATT-SG65i (in $0.1 \mathrm{M} \mathrm{NaCl}$ ) and (b) TTT CCC TTT CCC CCC-SG65i (in $0.1 \mathrm{M} \mathrm{NaCl}$ ) in PEG6kDa/DX250kDa, PEG2kDa/DX250kDa and PEG1.5kDa/DX250kDa ATP systems. Absorption spectrum of the final bottom fraction obtained after loading (c) TTA TAT TAT ATT-SG65i (in 0.1 M NaCl) and (d) TTT CCC TTT CCC CCC-SG65i (in $0.1 \mathrm{M} \mathrm{NaCl}$ ) in PEG6kDa/DX250kDa, PEG2kDa/DX250kDa and PEG1.5kDa/DX250kDa ATP systems. Absorption spectrum of (e) two (6,5) enantiomers obtained from the $(6,5)$ super sequence in PEG1.5kDa/DX250kDa ATP system. (f) two $(8,3)$ enantiomers obtained from the $(8,3)$ super sequence in PEG1.5kDa/DX250kDa ATP system. 


\section{Reduced Interfacial Trapping in PEG1.5kDa/DX250kDa ATP System}

Many DNA-SWCNT dispersions suffer from severe interfacial trapping in PEG/PAM ATP system. $(9,4)$ was obtained with SWCNT dispersion made with CTT CCC TTC. ${ }^{1}$ Figure S4a and S4b showed each fractions obtained in PEG/PAM and PEG1.5kDa/DX250kDa ATP system. In PEG/PAM system, after the extraction of a few top phases, severe interfacial trapping happens between the 5 th top phase (5T) and the 5th bottom phase (5B) marked by the red circle (Figure S4a), which is more obvious from the tile view. However, no interfacial trapping happens in PEG1.5kDa/DX250kDa ATP system (Figure S4b). The visible interface in the red circle of Figure S4b is due to the difference in refractive index between the top phase and bottom phase. It is not interfacial trapping. The tilt view clearly shows that no SWCNTs are trapped in the interface. Figure S4c lists the amount of PVP10kDa needed to extract the corresponding fraction during a $200 \mu \mathrm{L}$ scale separation. Although the purity of $(9,4)$ separated from the top phase in PEG1.5kDa/DX250kDa system is not as good as that from PEG/PAM system (Figure S4d), the last bottom fraction is highly enriched in $(6,6)$ while the last bottom fraction in PEG/PAM system is a mixture (Figure S4e). The higher stability of DNA-SWCNT dispersion in PEG1.5kDa/DX250kDa ATP system allows multi-step purification and increase the chance of obtaining fraction containing pure SWCNT from the final bottom phase. As shown in Figure S4f, for the sorting of (ATT) $)_{4}$-SG65i dispersion in PEG1.5kDa/DX250kDa ATP system, after the extraction of a few top phases, the final bottom phase is pure $(7,5)$. Previously, this sequence only works in (PEG+PEG-DA)/DX ATP system. $^{2}$ 
(a)

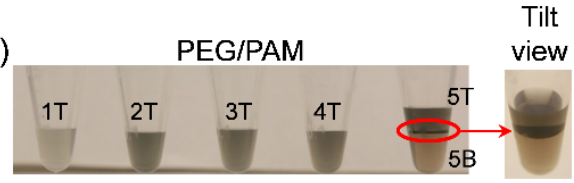

(b)

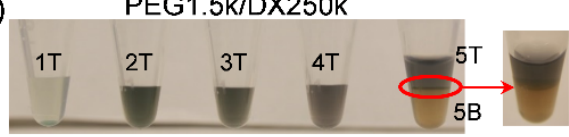

(d)

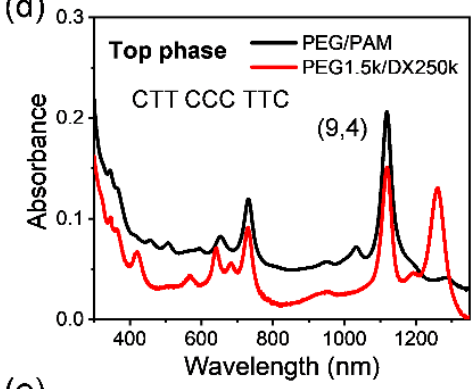

(e)

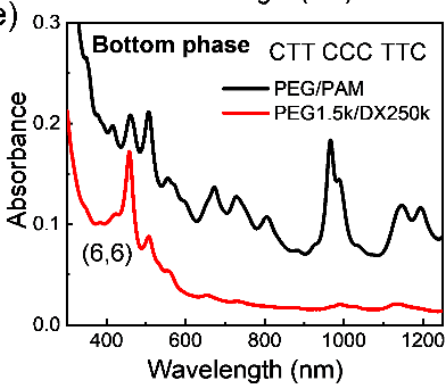

(c)

\begin{tabular}{|c|c|c|}
\hline \multirow{2}{*}{ Fraction } & \multicolumn{2}{|c|}{ Amount of PVP needed } \\
\hline & PEG/PAM & PEG1.5k/DX250k \\
\hline $1 \mathrm{~T}$ & & $0.5 \mu \mathrm{L} 5 \%$ PVP $10 \mathrm{k}$ \\
\hline $2 \mathrm{~T}$ & $1 \mu \mathrm{L} 5 \%$ PVP $10 \mathrm{k}$ & $1 \mu \mathrm{L} 20 \%$ PVP $10 \mathrm{k}$ \\
\hline 3T & $0.5 \mu \mathrm{L} 5 \%$ PVP $10 \mathrm{k}$ & $0.5 \mu \mathrm{L} 20 \%$ PVP $10 \mathrm{k}$ \\
\hline $4 \mathrm{~T}$ & $1 \mu \mathrm{L} 5 \%$ PVP $10 \mathrm{k}$ & $0.5 \mu \mathrm{L} 20 \%$ PVP $10 \mathrm{k}$ \\
\hline 5T & $2 \mu \mathrm{L} 1 \%$ PVP $10 \mathrm{k}$ & $1 \mu \mathrm{L} 10 \%$ PVP $10 \mathrm{k}$ \\
\hline
\end{tabular}

(f)

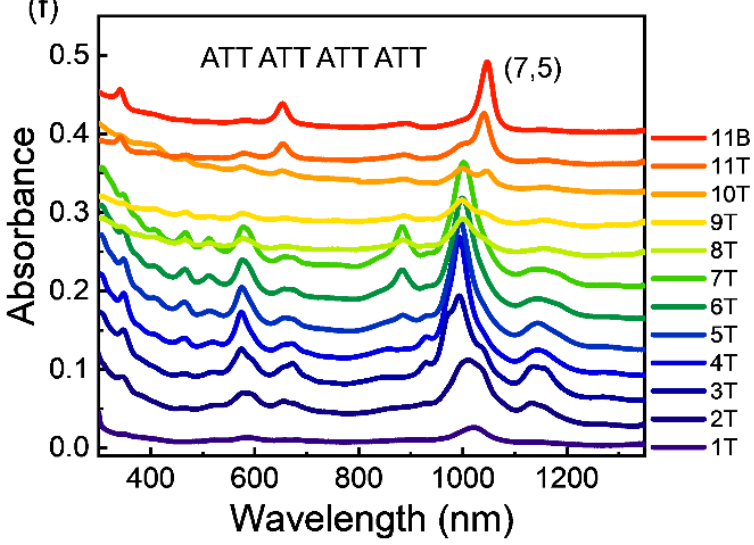

Figure S4. The picture of each fraction obtained when CTT CCC TTC-EG150X dispersion is loaded on (a) PEG/PAM and (b) PEG1.5kDa/DX250kDa ATP system. (c) The amount of PVP10kDa needed to extract the corresponding top fraction in part $\mathrm{a}$ and $\mathrm{b}$. Absorption spectrum of the (d) first top fractions and (e) final bottom fractions after loading CTT CCC TTC-EG150X dispersion in PEG/PAM and PEG1.5kDa/DX250kDa ATP system. (f) Absorbance spectra of each fractions obtained with continuous addition of PVP during the sorting of (ATT) $4^{-}$ SG65i (in $30 \mathrm{mM} \mathrm{NaCl}$ ) in PEG1.5kDa/DX250kDa ATP system. 


\section{Detailed Procedure of the Multi-Step Sorting of (TCTCCC) ${ }_{2}$ TCT-SG65i Dispersion}

Table S1. Detailed sorting procedure of (TCTCCC) ${ }_{2}$ TCT-SG65i dispersion at $20^{\circ} \mathrm{C}$

\begin{tabular}{|c|c|c|c|c|}
\hline step 1 & \multicolumn{4}{|c|}{ 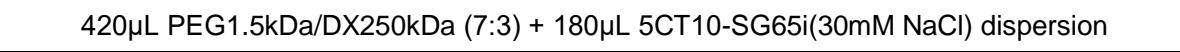 } \\
\hline step 2 & & & & $\begin{array}{c}\text { Vortex, centrifuge } \\
\text { then extract } 1 \mathrm{~T}\end{array}$ \\
\hline step 3 & $\begin{array}{c}+0.75 \mu \mathrm{L} \\
2 \% \text { PVP10kDa }\end{array}$ & & $\begin{array}{l}+300 \mu \mathrm{L} \\
\text { blank top phase }\end{array}$ & $\begin{array}{c}\text { Vortex, centrifuge } \\
\text { then extract } 2 \mathrm{~T}\end{array}$ \\
\hline step 4 & $\begin{array}{c}+1 \mu \mathrm{L} \\
10 \% \text { PVP10kDa }\end{array}$ & & $\begin{array}{l}+300 \mu \mathrm{L} \\
\text { blank top phase }\end{array}$ & $\begin{array}{c}\text { Vortex, centrifuge } \\
\text { then extract 3T }\end{array}$ \\
\hline step 5 & $\begin{array}{c}+1 \mu \mathrm{L} \\
30 \% \text { PVP10kDa }\end{array}$ & & $\begin{array}{c}+300 \mu \mathrm{L} \\
\text { blank top phase }\end{array}$ & $\begin{array}{c}\text { Vortex, centrifuge } \\
\text { then extract } 4 \mathrm{~T}\end{array}$ \\
\hline step 6 & $\begin{array}{c}+1.5 \mu \mathrm{L} \\
30 \% \text { PVP10kDa }\end{array}$ & & $\begin{array}{c}+300 \mu \mathrm{L} \\
\text { blank top phase }\end{array}$ & $\begin{array}{c}\text { Vortex, centrifuge } \\
\text { then extract } 5 \mathrm{~T}\end{array}$ \\
\hline step 7 & $\begin{array}{c}+1 \mu \mathrm{L} \\
30 \% \text { PVP10kDa }\end{array}$ & & $\begin{array}{l}+300 \mu \mathrm{L} \\
\text { blank top phase }\end{array}$ & $\begin{array}{c}\text { Vortex, centrifuge } \\
\text { then extract } 6 \mathrm{~T}\end{array}$ \\
\hline step 8 & $\begin{array}{c}+0.5 \mu \mathrm{L} \\
30 \% \text { PVP10kDa }\end{array}$ & & $\begin{array}{l}+300 \mu \mathrm{L} \\
\text { blank top phase }\end{array}$ & $\begin{array}{c}\text { Vortex, centrifuge } \\
\text { then extract } 7 \mathrm{~T}\end{array}$ \\
\hline step 9 & $\begin{array}{c}+0.75 \mu \mathrm{L} \\
20 \% \text { PVP10kDa }\end{array}$ & & $\begin{array}{l}+300 \mu \mathrm{L} \\
\text { blank top phase }\end{array}$ & $\begin{array}{c}\text { Vortex, centrifuge } \\
\text { then extract } 8 \mathrm{~T}\end{array}$ \\
\hline step 10 & $\begin{array}{c}+0.5 \mu \mathrm{L} \\
20 \% \text { PVP10kDa }\end{array}$ & & $\begin{array}{l}+300 \mu \mathrm{L} \\
\text { blank top phase }\end{array}$ & $\begin{array}{c}\text { Vortex, centrifuge } \\
\text { then extract 9T }\end{array}$ \\
\hline step 11 & & $\begin{array}{c}+0.5 \mu \mathrm{L} \\
1 \mathrm{M} \mathrm{pH}=7 \text { sodium phosphate buffer }\end{array}$ & $\begin{array}{l}+300 \mu \mathrm{L} \\
\text { blank top phase }\end{array}$ & $\begin{array}{l}\text { Vortex, centrifuge } \\
\text { then extract } 10 \mathrm{~T}\end{array}$ \\
\hline step 12 & $\begin{array}{c}+1 \mu \mathrm{L} \\
2 \% \text { PVP10kDa }\end{array}$ & $\begin{array}{c}+1.5 \mu \mathrm{L} \\
1 \mathrm{M} \mathrm{pH}=7 \text { sodium phosphate buffer }\end{array}$ & $\begin{array}{l}+300 \mu \mathrm{L} \\
\text { blank top phase }\end{array}$ & $\begin{array}{l}\text { Vortex, centrifuge } \\
\text { then extract } 11 \mathrm{~T}\end{array}$ \\
\hline step 13 & $\begin{array}{c}+0.5 \mu \mathrm{L} \\
5 \% \text { PVP10kDa }\end{array}$ & $\begin{array}{c}+2 \mu \mathrm{L} \\
1 \mathrm{M} \mathrm{pH}=7 \text { sodium phosphate buffer }\end{array}$ & $\begin{array}{l}+300 \mu \mathrm{L} \\
\text { blank top phase }\end{array}$ & $\begin{array}{l}\text { Vortex, centrifuge } \\
\text { then extract } 12 \mathrm{~T}\end{array}$ \\
\hline step 14 & $\begin{array}{c}+2.5 \mu \mathrm{L} \\
5 \% \text { PVP10kDa }\end{array}$ & $\begin{array}{c}+1 \mu \mathrm{L} \\
3 \mathrm{M} \mathrm{pH}=7 \text { sodium phosphate buffer }\end{array}$ & $\begin{array}{l}\quad+300 \mu \mathrm{L} \\
\text { blank top phase }\end{array}$ & $\begin{array}{c}\text { Vortex, centrifuge } \\
\text { then extract13T, } \\
\text { left } 13 \mathrm{~B}\end{array}$ \\
\hline
\end{tabular}

\section{The pH Dependence of C-rich Sequence}

Many C-rich sequence show better wrapping of SWCNTs under low $\mathrm{pH}$ conditions. This is reflected by the better resolution of their absorption spectrum under low $\mathrm{pH}$ conditions. The first and middle column of Figure S5 show the absorption and normalized absorption spectra of SWCNTs dispersed by different DNA sequences under different $\mathrm{pH}$ (or salt) condition. For GC11 sequence, the yield of the dispersion is very low in $\mathrm{NaCl}$. However, when $30 \mathrm{mM}$ sodium phosphate salt were used to make DNA-SWCNTs dispersion, the yield is greatly improved. Figure $S$ $5 \mathrm{~b}$ shows that the resolution of the normalized absorption spectrum gradually improved as the $\mathrm{pH}$ of sodium phosphate buffer switch from 8 to 6 and maintains at similar level as the $\mathrm{pH}$ further decrease to 4 . This is a common phenomenon for a lot of C-rich sequences we tested (Figure S 5e, S5h and S5k). However, when the pH further reduces to 3 , the resolution deteriorates. For $(11,0)$-recognition sequence $\left(\mathrm{GC}_{11}\right.$ and $\left.\mathrm{C}_{12}\right)$, the purity and yield of $(11,0)$ sorted from the first top fraction gradually improved as the $\mathrm{pH}$ switch from 6 to 4 , while no $(11,0)$ appears in 
the top phase when the $\mathrm{pH}$ is larger than 6 or lower than 4 (black trace in Figure S5f). The better sorting result under low $\mathrm{pH}$ condition seems to be unique for $(11,0)$. Similar sorting result are not observed for $(8,3)$ and $(9,4)$ recognition sequence (Figure S5i and S51).
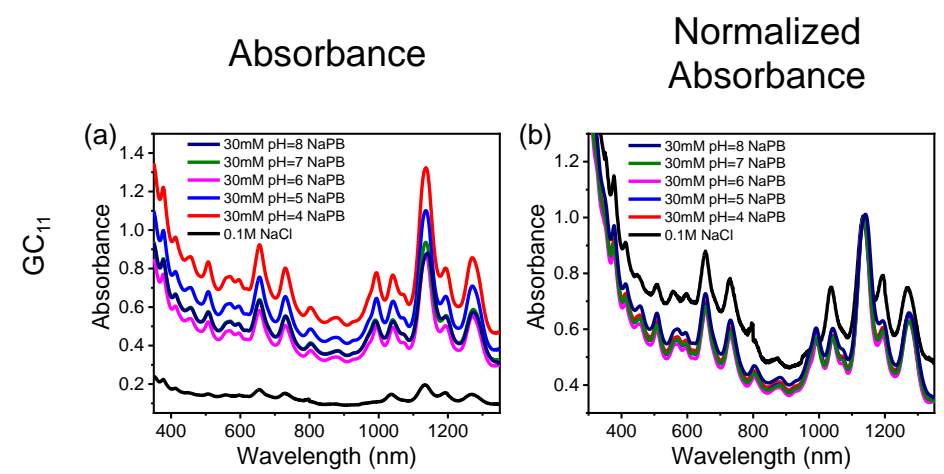

First Top Fraction
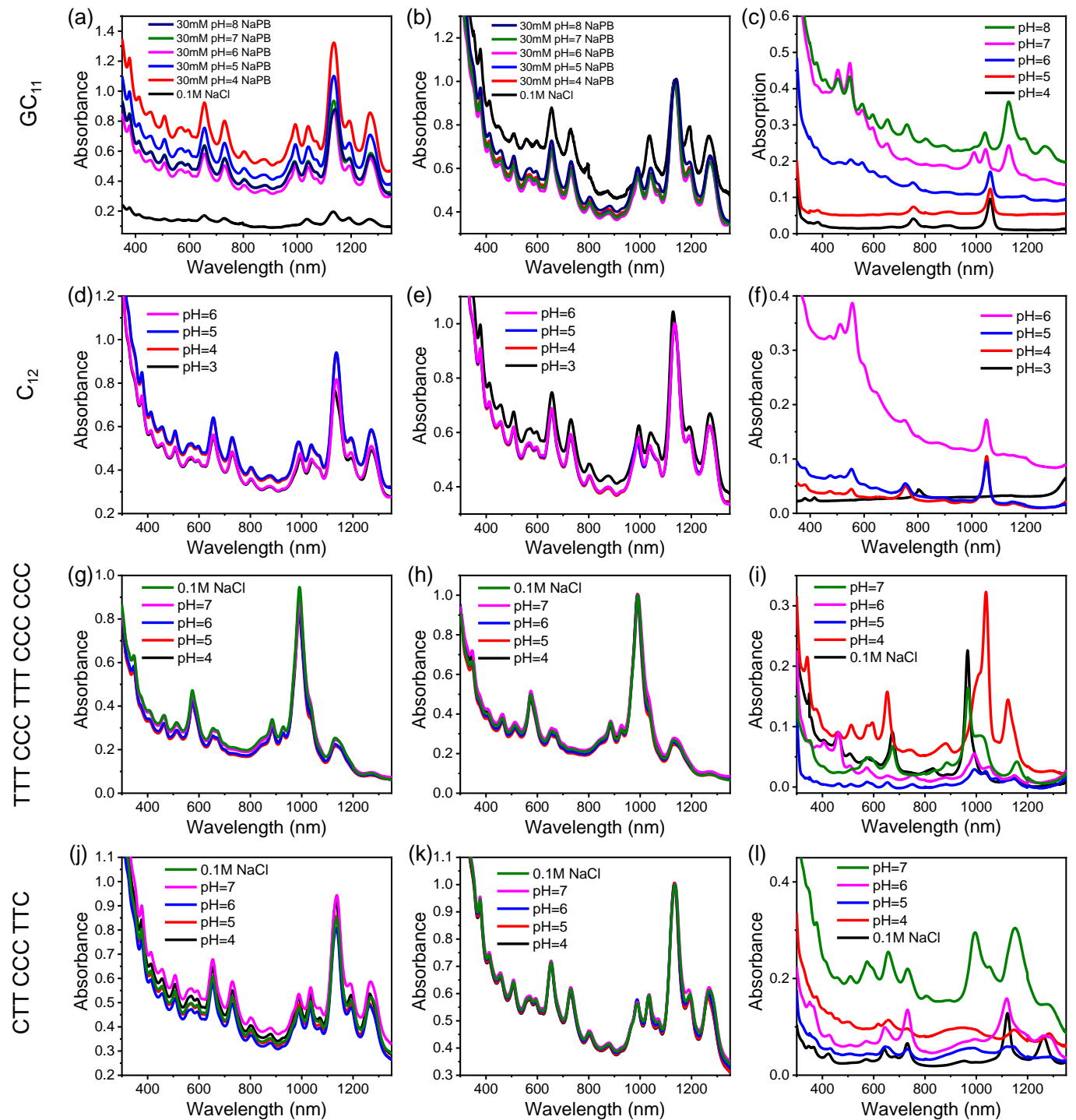

Figure S5. (a) Absorption and (b) normalized absorption spectrum of $\mathrm{GC}_{11}$-EG150x dispersion under different $\mathrm{pH}$ condition, (c) the first top fraction containing SWCNTs sorted from the corresponding dispersion in PEG1.5kDa/DX250kDa ATP system; (d) Absorption and (e) normalized absorption spectrum of $\mathrm{C}_{12}$-EG150x dispersion under different $\mathrm{pH}$ condition, (f) the first top fraction containing SWCNTs sorted from the corresponding dispersion in PEG1.5kDa/DX250kDa ATP system; (g) Absorption and (h) normalized absorption spectrum of TTT CCC TTT CCC CCC-SG65i dispersion under different $\mathrm{pH}$ condition, (i) the first top fraction containing SWCNTs sorted from the corresponding dispersion in PEG1.5kDa/DX250kDa ATP system; (j) Absorption and (k) normalized absorption spectrum of CTT CCC TTC-EG150x dispersion under different $\mathrm{pH}$ condition, (1) the first top fraction containing SWCNTs sorted from the corresponding dispersion in PEG1.5kDa/DX250kDa ATP system.

Fourier transform infrared spectroscopy (FTIR) of $\mathrm{GC}_{11}$-SG65i and $\mathrm{C}_{12}$-SG65i dispersions made in $30 \mathrm{mM} \mathrm{pH}=7$ and $\mathrm{pH}=4$ sodium phosphate solutions are shown in Figure $\mathrm{S} 6$. These spectra were obtained by first freeze-drying the dispersions and then measuring on a PerkinElmer Spectrum Spotlight 200 FT-IR microscopy between $4000 \mathrm{~cm}^{-1}$ and $650 \mathrm{~cm}^{-1}$. The 
carbonyl stretch vibration band at $1724 \mathrm{~cm}^{-1}$ is indicative of the protonation of cytidine. Upon lowing $\mathrm{pH}$ this band becomes stronger, indicating more cytidines undergo protonation. The carbonyl stretch of neutral cytidine appears at $1654 \mathrm{~cm}^{-1}\left(\mathrm{GC}_{11^{-}}\right.$ SG65i, Figure S6a) and $1658 \mathrm{~cm}^{-1}\left(\mathrm{C}_{12}-\mathrm{SG} 65 \mathrm{i}\right.$, Figure $\left.\mathrm{S} 6 \mathrm{~b}\right)$ at $\mathrm{pH}=7$. Under low $\mathrm{pH}$ condition, this band shows a blue shift to $1664 \mathrm{~cm}^{-1}$. This blue shift was attributed to the protonation of cytidine and hydrogen bonding formed between hemiprotonated $\mathrm{C}^{-} \mathrm{C}^{+}$base pairs, which results in a better localization of the $\pi$ electrons on the carbonyl groups and consequently in higher $\mathrm{C}=\mathrm{O}$ force constants and frequencies. Similar band shifts were observed in other experiment under appropriate $\mathrm{pH}$ conditions. ${ }^{3-11}$ The band around $1490 \mathrm{~cm}^{-1}$ was assigned to motions involving $\mathrm{C}_{4}, \mathrm{~N}_{4}$ and $\mathrm{N}_{3}$ atoms. The bands between $1200 \mathrm{~cm}^{-1}$ and $900 \mathrm{~cm}^{-1}$ were associated with vibrations of groups in the sugar and phosphate part of DNA. ${ }^{12}$
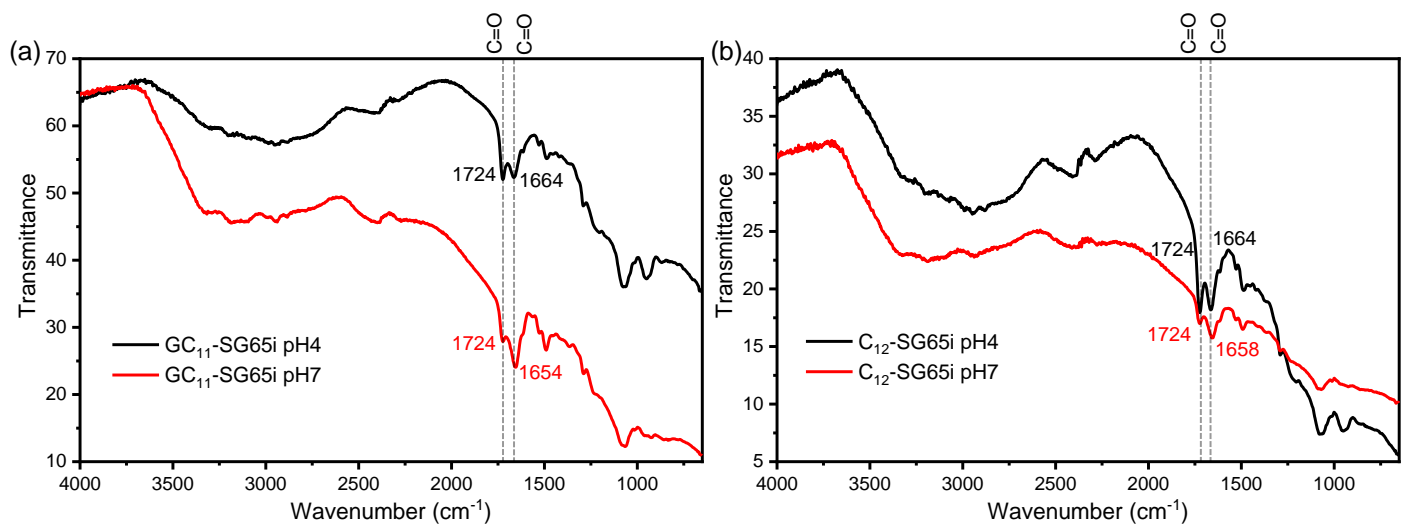

Figure S6. FTIR spectroscopy of (a) GC11-SG65i dispersion made in $30 \mathrm{mM} \mathrm{pH=7}$ (red) and pH=4 (black) sodium phosphate buffers and (b) C12-SG65i dispersion made in $30 \mathrm{mM} \mathrm{pH=7} \mathrm{(red)} \mathrm{and} \mathrm{pH}=4$ (black) sodium phosphate buffers.

\section{References}

(1) Ao, G.; Streit, J. K.; Fagan, J. A.; Zheng, M. Differentiating Left-and Right-handed Carbon Nanotubes by DNA. J. Am. Chem. Soc. 2016, 138, 16677-16685.

(2) Ao, G. Y.; Khripin, C. Y.; Zheng, M. DNA-Controlled Partition of Carbon Nanotubes in Polymer Aqueous Two-Phase Systems. J. Am. Chem. Soc. 2014, 136, 10383-10392.

(3) Petrovic, A. G.; Polavarapu, P. L. Structural Transitions in Polyribocytidylic Acid Induced by Changes in $\mathrm{pH}$ and Temperature: Vibrational Circular Dichroism Study in Solution and Film States. J. Phys. Chem. B 2006, 110, 22826-22833.

(4) Panin, L. E.; Kunitsyn, V. G.; Tuzikov, F. V. Changes in the Secondary Structure of Highly Polymeric DNA and CC(GCC)n-Type Oligonucleotides under the Action of Steroid Hormones and Their Complexes with Apolipoprotein A I. J. Phys. Chem. B 2006, 110, 13560-13571.

(5) Maleev, V.; Semenov, M.; Kashpur, V.; Bolbukh, T.; Shestopalova, A.; Anishchenko, D. Structure and hydration of polycytidylic acid from the data of infrared spectroscopy, EHF dielectrometry and computer modeling. J. Mol. Struct. 2002, 605, 51-61.

(6) Geinguenaud, F.; Liquier, J.; Brevnov, M. G.; Petrauskene, O. V.; Alexeev, Y. I.; Gromova, E. S.; Taillandier, E. Parallel Self-Associated Structures Formed by T,C-Rich Sequences at Acidic pH. Biochemistry 2000, 39, 12650-12658.

(7) Gavira, J. M.; Campos, M.; Diaz, G.; Hernanz, A.; Navarro, R. Vibrational analysis and 
spectra of cytidine 3' -monophosphate (3' -CMP). Vib. Spectrosc. 1997, 15, 1-16.

(8) Florián, J.; Baumruk, V.; Leszczyński, J. IR and Raman Spectra, Tautomeric Stabilities, and Scaled Quantum Mechanical Force Fields of Protonated Cytosine. J. Phys. Chem. 1996, 100, 5578-5589.

(9) Liquier, J.; Letellier, R.; Dagneaux, C.; Ouali, M.; Morvan, F.; Raynier, B.; Imbach, J. L.; Taillandier, E. Triple helix formation by. alpha.-oligodeoxynucleotides: A vibrational spectroscopy and molecular modeling study. Biochemistry 1993, 32, 10591-10598.

(10) Zundel, G.; Lubos, W. D.; Kölkenbeck, K. Proton dispersion forces. Secondary-structure stabilizing forces between the hydrogen bonds of the polynucleotides. Biophys. J. 1972, 12, 1509-1514.

(11) Hartman, K. A.; Rich, A. The Tautomeric Form of Helical Polyribocytidylic Acid. J. Am. Chem. Soc. 1965, 87, 2033-2039.

(12) Bradbury, E. M.; Price, W. C.; Wilkinson, G. R. Infrared studies of molecular configurations of DNA. J. Mol. Biol. 1961, 3, 301-317. 